\title{
Mold allergy, its prevention and therapy - part 2
}

\begin{abstract}
In the current treatment of mold allergy, there are two modalities: Preventive and therapeutic approaches. Preventive approaches include measurements against indoor as well as outdoor molds. Relative humidity is suggested to maintain at $<50 \%$ indoor environments. Windows are kept closed in warm months and air-conditioning ought to be used. Indoor plants, as a possible cause of allergen, should be exposed to direct sunlight from time to time.

Visible molds are cleaned and mold-infested resources are should be discarded from houses Washable surfaces must be cleaned with 5\% bleach and detergent solution. Damp carpets are ought to dried out quickly and to think taking them away if humidity is not immediately goes away. In bathrooms, curtains and tile should be regularly checked and mended to keep waterproof. Firewood or newspaper piles or old books are regularly scrutinized and thrown away as necessary. Drip pans for fridges, garages and basements at homes should be regularly checked and cleaned. Water leaks in dwellings should be fixed swiftly.

In outdoor environment, intense exposure to moldy plants must be avoided. A well-fitted particulate mask when functioning with moldy objects ought to be used. Air-conditioned vehicles should be utilized. Therapeutic approaches mentioned here for fungal allergy is specifically mold allergen immunotherapy in addition to pharmacotherapy against the effects of mold allergy. Mold allergen immunotherapy, as a specific therapy of fungal allergy, is previously hindered by the poor quality of most of the commercial extracts. There have been only several studies evaluating the efficacy of allergen immunotherapy with Alternaria extracts in patients with respiratory mold allergy.

A meta-analysis demonstrated that clinical efficacy of allergen immunotherapy with mold extracts was revealed in 79 actively treated cases in 4 controlled trials, with only 2 mold species, namely Alternaria alternate and Cladosporium herbarium. Recently the American Academy of Allergy, Asthma \& Immunology concluded that immunotherapy might be effective for mold allergy. In near future, diagnosis and therapy for mold allergy will be more decisive and effective with newly developed production techniques and methods.
\end{abstract}

Keywords: mold allergy, allergic rhinitis, asthma, prevention, immunotherapy, slit, clinical immunology, sublingual immunotherapy, treatment, allergy, ige, ait, sublingual
Volume 2 Issue 5 - 2015

\author{
Oner Ozdemir \\ Department of Pediatrics, Research and Training Hospital of \\ Sakarya University, Turkey
}

Correspondence: Öner Özdemir, Division of Allergy and Immunology, Department of Pediatrics, Research and Training Hospital of Sakarya University, Faculty of Medicine, Sakarya University, Adnan Menderes Cad, Sa $\square$ lık Sok No: 195, Adapazari, Sakarya, Turkey, Tel 90-264-444-54-00, Fax 90-264-275-91-92,Email ozdemir_oner@hotmail.com

Received: October 25, 2015 | Published: October 30, 2015

\section{Introduction}

In the first part of this article; we firstly described molds as indoor and outdoor allergens, then mechanisms of mold allergen-induced sensitization, sensitization prevalence and manifestations of mold allergy in upper and lower respiratory systems were told. In this second part, preventive / therapeutic approaches for mold allergy as well as new diagnostic / therapeutic models and future expectations will be told.

In the current treatment of mold allergy, there are two modalities: Preventive and therapeutic approaches. Preventive approaches include measurements against indoor as well as outdoor molds. Therapeutic approaches mentioned here for fungal allergy is specifically mold allergen immunotherapy (AIT) in addition to pharmacotherapy against the effects of mold allergy.

\section{Preventive measurements against mold allergy}

Indoor molds Relative humidity is suggested to maintain at $<50 \%$ indoor environments. Windows are kept closed in warm months and air-conditioning ought to be used. Indoor plants, as a possible cause of allergen, should be exposed to direct sunlight from time to time. Visible molds are cleaned and mold-infested resources are should be discarded from houses. Washable surfaces must be cleaned with 5\% bleach and detergent solution. ${ }^{1}$ Damp carpets are ought to dried out quickly and to think taking them away if humidity is not immediately goes away. In bathrooms, curtains and tile should be regularly checked and mended to keep waterproof. Firewood or newspaper piles or old books are regularly scrutinized and thrown away as necessary. Drip pans for fridges, garages and basements at homes should be regularly checked and cleaned. Water leaks in dwellings should be fixed swiftly.

Outdoor molds: In outdoor environment, intense exposure to moldy plants must be avoided. A well-fitted particulate mask when functioning with moldy objects ought to be used. Air-conditioned vehicles should be utilized. ${ }^{1-3}$

\section{Therapeutic approaches for mold allergy: mold allergen immunotherapy}

Mold AIT, as a specific therapy of fungal allergy, is previously hindered by the poor quality of most of the commercial extracts. In 1980 's, fungal extracts in AIT mixes were thought to be responsible for the most common and severe side effects in 3 patients having Alternaria iridis/alternate and 8 patients with Cladosporium herbarium allergy. ${ }^{4}$ Later studies demonstrated much better tolerance to fungal extracts, possibly because better-quality standardized AIT extracts were utilized. ${ }^{5}$

\section{Rules of mold allergen immunotherapy}

There were conflicting reports for the ability of some mold extracts to degrade pollens or epithelial extracts. The discrepancies are probably due to lot and industrialized variations. Combinations 
of pollen extracts with fungal extracts for AIT is not suggested if not both are utilized directly after preparation of the mix. Grass pollen extracts are very vulnerable and ragweed extracts are comparatively resistant to the effects of proteases. Alternaria from mold extracts had the most effective one in degrading pollens. ${ }^{5}$ In mixing AIT extracts, the best way is to not blend extracts of pollens, house dust mites, or animal dander's with those having protease activity such as molds. Similarly, the 2003 update to the AIT Practice Parameters suggested that fungal extracts should not be mixed with pollens, not including ragweed. Following Practice Parameter updates conceded that inconsistent data existed on the durability of ragweed when assorted with molds and suggested that all pollen should be kept apart from high-protease-containing extracts. It is perhaps fair enough to unite protease- containing extracts with each other. However, some other reviews suggested that mixing fungal extracts ought to avoid..$^{-10}$

\section{Subcutaneous allergen immunotherapy (SC-AIT) for Alternaria and Cladosporium}

Studies of SC-AIT with Alternaria extracts there have been only several studies evaluating the efficacy of AIT with Alternaria extracts in patients with respiratory mold allergy. A study in 1982 firstly showed that 7 out of $38(19 \%)$ children desensitized with Alternaria extract discontinued the AIT due to serious side effects. ${ }^{4}$ In a study by Cantani et al from Italy, 39 children with Alternaria-induced asthma/ rhinitis were immunized with specific Alternaria extracts and were observed for 3years. The investigators demonstrated that AIT with Alternaria was effective in reducing symptoms and medication needs in $80 \%$ of the children getting cumulative doses of 80,000 protein nitrogen units or more. The authors in this prospective case-control study of AIT with Alternaria reported no systemic reactions but mild local reactions. ${ }^{11}$

A more recent study used AIT in 24 (5-56years of age) patients only allergic to Alternaria. Horst et al. utilized 2-day rush protocol; maintenance therapy was applied for 1year. They showed that AIT with a standardized Alternaria extract was successful in these patients. AIT resulted in lesser symptom- medication scores, augmented mean challenging dose of Alternaria required for a positive nasal challenge, and decreased skin test reaction. ${ }^{12}$

In 2000, 129 patients treated with standardized commercial extracts of Alternaria (3892 doses), Tabar et al demonstrated that only 76 out of $3892(1.95 \%)$ injections caused to adverse reactions, which observed in $39.5 \%$ of the patients. Tolerance to Alternaria mold extracts was worse than for any other allergenic extracts, although most reactions developed were mild. These patients were with asthma and/or rhinitis induced by Alternaria allergy. In all patients a biologically standardized Alternaria tenuis depot extract including $5 \mathrm{BU} / \mathrm{ml}$ was utilized consistent with a conventional AIT program. In this study, a group of 46 (5-14years of age) children had more frequent adverse effects, among the 129 patients enrolled in the study. In this young age group more systemic reactions were observed with the AIT beginning dose, though not with the maintenance doses when compared with the adults.

Tabar et al., ${ }^{13}$ did not observe any differences in ocular and nasal symptoms between groups taken AIT or placebo. In the latest study from the same group, side effects were reported after only $0.28 \%$ of all injections. Systemic adverse reactions were seen in 1 subject. Local side effects were also unusual ( $1.11 \%$ of doses given), were temporary, and did not need rescue treatment. Hence they concluded that AIT with a standardized Alternaria extract is harmless in children. ${ }^{14}$ Children having allergic rhinitis/asthma allergic to Alternaria utilized mold AIT or placebo for 3years in a study by Kuna et al. Symptom and medication scores were notably decreased in the AIT group following the 2 nd and 3rdyears, with a decline in the symptom scores by $63 \%$ after AIT. The AIT group had a significant decline in symptoms after a single dose nasal allergen challenge compared to their baseline and to the placebo group one year after completion of the 3year AIT. ${ }^{15}$

The children were randomized by Martínez-Cañavate Burgos et al to either an active group (22 patients) given Alternaria immunotherapy with cluster regimen and symptomatic treatment, or to a control group (19 patients) getting only symptomatic treatment. Follow-up contained the evaluation of fungal extract tolerance, the symptom and medication score, and possible alterations in the subsequent in vitro parameters: specific IgE, specific IgG4 and interleukins (IL-2/-4/-5/$10 /-13$ and IFN- $\gamma$ ). The SEICAP immunotherapy group carried out this multicenter tolerance study in pediatric patients, in which good tolerance of the extract was seen - with a low percentage of side effects $(0.95 \%$ incidence of both local and systemic reactions at the dose applied). ${ }^{16}$

Studies of SC-AIT with Cladosporium extracts Malling et al., ${ }^{17}$ demonstrated 3 anaphylactic reactions with Cladosporium allergen extracts administered in adults; moreover, asthma worsened in all AIT patients. Local side effects were shown in $70 \%$ of patients. In another study of AIT against Cladosporium allergy, Dreborg et al did not detect any affects on symptom scores but see a decrease in medication scores. ${ }^{18}$ In a placebo-controlled, double-blind study using high-dose $(100,000 \mathrm{BU})$ allergen extracts; $81 \%$ of the Cladosporium herbarium -allergic patients desensitized with Cladosporium herbarium extract reported improvement, while $19 \%$ worsening in their clinical symptoms. ${ }^{19}$

A meta-analysis of SC-AIT:A meta-analysis by Helbling et al demonstrated that clinical efficacy of AIT with mold extracts was revealed in 79 actively treated cases in 4 controlled trials, with only 2 mold species, namely Alternaria alternata and Cladosporium herbarium (7).

Sublingual immunotherapy (SLIT) for Alternaria:Patients with rhinitis \pm intermittent asthma and allergy to Alternaria alternata were enrolled. After a baseline season, SLIT or placebo was given for 10months. Twenty-seven (14-42years of age) patients were randomized, and 26 finished the study. After treatment, patients receiving SLIT had a considerable improvement in symptoms and a reduction in medication scores vs. placebo and vs. the run-in season. SPT reactivity significantly decreased only in the SLIT group. No change was seen in specific IgG4 levels in the both groups, whereas specific IgE levels against Alt a1 significantly increased in the active group. ${ }^{20}$

A randomized, parallel group study in 52 subjects $(32 \mathrm{men}$, mean age: $20 \pm 9$ years) with clinically- and laboratory-confirmed Alternaria alternata respiratory allergies (allergic rhinitis \pm asthma) was performed. Patients (n:34) were randomized to SLIT treatment for 3 consecutive years or to a control group (n:18). After 3 years, the visual analogue scale score was significantly increased as $4.7 \pm 0.8$ in the SLIT group and $2 \pm 1.6$ in the control group. Clinical improvement was observed in 33/ 34 subjects in the SLIT group (97\%) and in $5 / 18(27 \%)$ in the control group. The medication score significantly decreased in the SLIT group. ${ }^{21}$ No serious side effects were observed and 3-year course of SLIT is found to be efficient and well-tolerated in the patients with respiratory allergy induced by Alternaria.

A recent study of SLIT with Alternaria extract in childhood asthma assessed clinical safety/efficacy and effects on in vivo and in vitro parameters. 19 patients given SLIT and another 19 received 
symptomatic treatment. The initial phase of SLIT lasted 3 months until the maximum dose was reached. This was maintained for 12 months; the mean cumulative dose was 280,000 PNU. Important variations such as in reduction in the symptom-medication score in the actively treated group after 12months of SLIT, when compared with the control group. SLIT with Alternaria extract was well tolerated with 0.42 adverse reactions per 100 doses given. ${ }^{22}$

\section{Comparison of injective (SC-AIT) vs. SLIT in Alternaria alternata allergic patients}

Purified and standardized extract of Alternaria alternata was given to 23 patients for 2years. Excellent tolerance was shown to SLIT, while 4 (2 medium-grade) side effects appeared during SC-AIT. This current study has also shown similar effectiveness of SLIT compared with conventional SC-AIT in patients treated with Alternaria alternata extracts. Clinical symptom and medication scores before and after the therapy improved, verifying an increase in the specific nasal challenge dose. $^{23}$

\section{Limitations to immunotherapy}

The role of AIT in allergies induced by molds is still not apparent in children at the moment. Mold AIT has been considered as unsuccessful with unstandardized extracts before $1990 .{ }^{24}$ Lack of standardized allergenic extracts hinders the treatment of Alternaria sensitivity. Most of the studies were done with variable allergenic extract content and potency. ${ }^{5}$ Although mold AIT is currently feasible in most countries, it is not suggested due to extract standardization problems and frequent side effects. Moreover, the utilization of fungal extracts in AIT is hindered by enormous fungal species and the lack of information on the degree of exposure to many molds. ${ }^{25}$ There is an absence of commercial extracts for many of the frequent fungi due to not growing on artificial media. ${ }^{26}$ Published studies have also major limitations, including lack of placebo control, open design, utilization of unstandardized extracts, short follow-up time, or not have of a baseline observation periods. ${ }^{15}$

There is a lack of randomized placebo-controlled trials when evaluating the efficacy of mold AIT with trials only focusing on AIT against Alternaria and Cladosporium. The 1993 European Academy of Allergology and Clinical Immunology (EAACI) position statement did not advise AIT with mold extracts for children, although more recently the American Academy of Allergy, Asthma \& Immunology (AAAAI) concluded that immunotherapy might be effective for mold allergy. ${ }^{27,28}$ The concerns raised by the EAACI included not only the lack of efficacy but also safety issues. A limited number of AIT studies performed in Europe with extracts of Cladosporium and Alternaria have demonstrated therapeutic efficacy. ${ }^{15}$ Cochrane reviews included just 2 out of 88 AIT trials with Cladosporium in its meta-analysis. ${ }^{29-31}$ There has been acceptable evidence showing Alternaria SCIT is beneficial in allergic children and improves medication scores, combined symptom-medication scores, and quality of life in the patients. ${ }^{32}$ Some evidence for Alternaria SLIT efficacy is appearing. ${ }^{33}$

Given these restrictions, it is suggested that mold AIT is limited to patients having allergy to a fungus, with symptoms taking place during times of high atmospheric exposure to that fungus, and in whom pharmacotherapy or prevention is not enough to manage symptoms.

\section{New diagnostic/therapeutic models and future expectations:}

Presently diagnosis of Alternaria alternata sensitization with natural as well as recombinant Alt a 1 allergens could be done. Alt a 1 , either in its natural or recombinant form, is sufficient for a dependable diagnosis of Alternaria alternata sensitivity or stimulates skin prick reactivity comparable with that formed by Alternaria alternata extract. ${ }^{34}$ Mold AIT was administered successfully for allergic fungal sinusitis. ${ }^{35,36}$ In a recent study, 4 patients having sino-nasal polyposis taken care of by mold AIT against Candida albicans have been described. The cases are treated either by subcutaneous injections (SC-AIT) or sublingual (SLIT) drops. ${ }^{37}$ In near future, diagnosis and therapy for mold allergy will be more decisive and effective with newly developed production techniques and methods.

\section{Acknowledgments}

None.

\section{Conflicts of interest}

Author declares there are no conflicts of interest.

\section{Funding}

None.

\section{References}

1. Bush RK, Prochnau JJ. Alternaria-induced asthma. J Allergy Clin Immunol . 2004;113(2):227-234.

2. Bush RK. Indoor allergens, environmental avoidance, and allergic respiratory disease. Allergy Asthma Proc . 2008;29(6): 575-579.

3. Wright LS, Phipatanakul W. Environmental remediation in the treatment of allergy and asthma: latest updates. Curr Allergy Asthma Rep 2014;14(3):419.

4. Kaad PH, Ostergaard PA. The hazard of mould hypo sensitization in children with asthma. Clin Allergy. 1982;12(3):317-320.

5. Nelson HS. The use of standardized extracts in allergen immunotherapy. J Allergy Clin Immunol. 2000;106(1 Pt 1):41-45

6. Grier TJ, LeFevre DM, Duncan EA, et al. Stability of standardized grass, dust mite, cat, and short ragweed allergens after mixing with mold or cockroach extracts. Ann Allergy Asthma Immunol . 2007;99(2):151-160.

7. Helbling A, Reimers A. Immunotherapy in fungal allergy. Curr Allergy Asthma Rep. 2003;3(5):447-453.

8. Nelson HS. Allergen immunotherapy: where is it now? J Allergy Clin Immunol. 2007;119(4):769-779.

9. Nelson HS, Ikle D, Buchmeier A. Studies of allergen extract stability: the effects of dilution and mixing. J Allergy Clin Immunol. 1996;98(2):382-388.

10. Coop CA. Immunotherapy for mold allergy. Clin Rev Allergy Immuno .2014;47(3):289-298

11. Cantani A, Businco E, Maglio A. Alternaria allergy: a three-year controlled study in children treated with immunotherapy. Allergol Immunopathol (Madr). 1988;16(1):1-4.

12. Horst M, Hejjaoui A, Horst V, et al. Double-blind, placebo-controlled rush immunotherapy with a standardized Alternaria extract. J Allergy Clin Immunol. 1990;85(2):460-472.

13. Tabar AI, Lizaso MT, García BE, et al. Tolerance of immunotherapy with a standardized extract of Alternaria tenuis in patients with rhinitis and bronchial asthma. J Investig Allergol Clin Immunol 2000;10(6):327-333.

14. Tabar AI, Lizaso MT, García BE, et al. Double-blind, placebo-controlled study of Alternaria alternata immunotherapy: clinical efficacy and safety. Pediatr Allergy Immunol . 2008;19(1):67-75

15. Kuna P, Kaczmarek J, Kupczyk M. Efficacy and safety of immunotherapy for allergies to Alternaria alternata in children. J Allergy Clin Immunol. 2011;127(2):502-508.e1-6. 
16. Martínez-Cañavate Burgos A, Valenzuela-Soria A, Rojo-Hernández A Immunotherapy with Alternaria alternata: present and future. Allergol Immunopathol . 2007;35(6):259-263.

17. Malling HJ, Dreborg S, Weeke B. Diagnosis and immunotherapy of mould allergy. V. Clinical efficacy and side effects of immunotherapy with Cladosporium herbarium. Allergy. 1986;41(7): 507-519.

18. Dreborg S, Agrell B, Foucard T, et al. A double-blind, multicenter immunotherapy trial in children, using a purified and standardized Cladosporium herbarium preparation. I. Clinical results. Allergy . 1986;41(2):131-140.

19. Malling HJ. Diagnosis and immunotherapy of mould allergy. With special reference to Cladosporium herbarium. Dan Med Bull. 1990;37(1):12-22.

20. Cortellini G, Spadolini I, Patella V, et al. Sublingual immunotherapy for Alternaria-induced allergic rhinitis: a randomized placebo-controlled trial. Ann Allergy Asthma Immunol. 2010;105(5):382-386.

21. Pozzan M, Milani M. Efficacy of sublingual specific immunotherapy in patients with respiratory allergy to Alternaria alternata: a randomised, assessor-blinded, patient-reported outcome, controlled 3-year trial. Curr Med Res Opin. 2010;26(12):2801-2806.

22. Criado Molina A, Guerra Pasadas F, Daza Muñoz JC, et al [Immunotherapy with an oral Alternaria extract in childhood asthma. Clinical safety and efficacy and effects on in vivo and in vitro parameters]. Allergol Immunopathol (Madr). 2002;30(6):319-330.

23. Bernardis P, Agnoletto M, Puccinelli P, et al. Injective versus sublingual immunotherapy in Alternaria tenuis allergic patients. J Investig Allergol Clin Immunol . 1996;6(1):55-62.

24. Esch RE. Manufacturing and standardizing fungal allergen products. $J$ Allergy Clin Immunol. 2004;113(2):210-215.

25. Simon-Nobbe B, Denk U, Pöll V, et al. The spectrum of fungal allergy. Int Arch Allergy Immunol. 2008;145(1):58-86.

26. Chapman MD, Ferreira F, Villalba M, et al. CREATE consortium The European Union CREATE project: a model for international standardization of allergy diagnostics and vaccines. $J$ Allergy Clin Immunol. 2008;122(5):882-889.e2.
27. Position paper: Immunotherapy with hymenoptera venoms. (EAACI) The European Academy of Allergology and Clinical Immunology. Allergy. 1993;48(14 suppl):36-46.

28. Joint Task Force on Practice Parameters; American Academy of Allergy, Asthma and Immunology; American College of Allergy, Asthma and Immunology; Joint Council of Allergy, Asthma and Immunology. Allergen immunotherapy: a practice parameter second update. $J$ Allergy Clin Immunol. 2007;120 (3 Suppl):S25-S85.

29. Abramson MJ, Puy RM, Weiner JM. Allergen immunotherapy for asthma. Cochrane Database Syst Rev. 2000;(2): CD001186.

30. Abramson MJ, Puy RM, Weiner JM. Allergen immunotherapy for asthma. Cochrane Database Syst Rev. 2003;4:CD001186.

31. Abramson MJ, Puy RM, Weiner JM. njection allergen immunotherapy for asthma. Cochrane Database Syst Rev. 2010;(8):CD001186.

32. Larenas-Linnemann DE, Pietropaolo-Cienfuegos DR, Calderón MA. Evidence of effect of subcutaneous immunotherapy in children: complete and updated review from 2006 onward. Ann Allergy Asthma Immunol. 2011;107(5):407-416.e11.

33. Larenas-Linnemann D, Blaiss M, Van Bever HP, et al. Pediatric sublingual immunotherapy efficacy: evidence analysis, 2009-2012. Ann Allergy Asthma Immunol. 2013;110(6):402-415.e9.

34. Twaroch TE, Focke M, Fleischmann K, et al. Carrier-bound Alt a 1 peptides without allergenic activity for vaccination against Alternaria alternata allergy. Clin Exp Allergy. 2012;42(6):966-975.

35. Hall AG, deShazo RD. Immunotherapy for allergic fungal sinusitis. Curr Opin Allergy Clin Immunol. 2012;12(6):629-634.

36. Mabry RL, Mabry CS. Immunotherapy for allergic fungal sinusitis: the second year. Otolaryngol Head Neck Surg. 1997;117:367-371.

37. Bénoliel P. [Treatment of sino-nasal polyposis by Candida albicans immunotherapy: apropos of 4 cases]. Allerg Immunol (Paris). 2001;33(10):388-394. 